\title{
Stochastic touristic walk: analytical approaches, algorithm and numerical results
}

\author{
Juliana Militão Berbert ${ }^{1}$, Rodrigo Silva González² and Alexandre Souto Martinez ${ }^{3}$
}

Manuscript received on September 23, 2008 / accepted on November 10, 2008

\begin{abstract}
Consider $N$ points randomly and uniformly distributed in a $d$-dimensional hypercube. A walker explores this disordered medium going to the nearest site, which has not been visited in the last $\mu$ (memory) steps. The walker trajectory is composed of a transient part and a periodic part (cycles). In this case, travelers can or cannot explore all available space, given rise to a crossover at critical memory, for one-dimensional systems $\mu_{1}=\ln _{2} N$, between localized and extended regimes. The deterministic rule can be softened to consider more realistic situations with the inclusion of a stochastic parameter $T$ (temperature). In this case, the walker movement is defined by a probability density function (PDF) that is parameterized by $T$ and a cost function, which increases as the distance among sites increases. As the temperature increases, the walker can escape from cycles and extend the exploration. Here we review the analytical results obtained for a system with arbitrary dimensionality $d$ for $\mu=0$ and one-dimensional systems with $\mu=1$. Also we suggest an extension of this system to study the influence of the temperature on the critical memory.
\end{abstract}

Keywords: stochastic walk, deterministic walk, non-Markovian processes, glass transition.

2E-mail: rsgonzalez@pg.ffclrp.usp.br

3E-mail: asmartinez@usp.br 


\section{INTRODUCTION}

Random walks and stochastic techniques have become nearly ubiquitous in science and engineering, specially in studies of natural phenomena. The random walks have had their origin in the analysis of the particles movement in fluids, as a dust particle over a water drop. One can observe the employment of the random walks implemented over a regular lattice or on disordered systems. A classical example is the Travelling Salesman Problem (TSP), where a walker (salesman) knows a priori the location of all sites (cities) in a disordered landscape. This salesman starts his walk in a given city and must visit all other cities once and return to the first one through the shortest path.

Being a non-polynomial (NP) hard problem, analytical and numerical studies are notably complicated. Nowadays, the most common numerical methods used to deal with this problem are simulated annealing and genetic algorithm. Although these methods are consolidated, they spend several computational effort. So, alternative methods, which reduces the computational effort, are desirable.

Lima et al. [1], in 2001, have introduced a new way to explore disordered systems, with a deterministic walker which moves having memory $\mu$. The walk was recognized as "Tourist Walk" (TW) [2] and, due to its memory, it is partially self-avoiding. Differently of TSP, in TW, the walker have to know only local information to guarantee a good exploration. Following the deterministic rule of going to the nearest site which has not been visited in the last $\mu$ steps, it has been found that even with low memory the walker may explore the whole system. The minimal memory that enable an extended exploration in one-dimensional systems is the critical memory $\mu_{1}=\ln _{2} N$. A stochastic version of this walk were investigated by Martinez et al. [3] on systems with arbitrary dimensionality $d$ and walker with $\mu=0$. RisauGusman et al. [4] have examined this stochastic walk over onedimensional systems and walker with $\mu=1$. In both cases a glass transition has been found. This article shows a review of the analitycal results obtained by [3] and [4] in the Section 2. Besides, numerical results for one-dimensional systems and walkers with $\mu \geq 0$ are shown in Section 3 .

\section{STOCHASTIC TOURIST WALK}

The stochastic tourist walk (STW) is a generalization of the deterministic tourist walk (DTW) [1], to comprehend more realistic situations. Consider a random walk on a disordered landscape composed of $N$ sites representing, for example, localized feeding sites as flowers, trees, water holes, islands, etc [3]. The disor- dered medium can be defined with each site coordinate, $x_{i}^{(k)}$, $i=1,2, \ldots, N$ and $k=1,2, \ldots, d$, being drawn in accordance with a random and uniform distribution along the unitary edges in a $d$-dimensional hypercube. To hop from one site to another, the walker uses a strategy based in some arbitrary cost function $E\left(D_{j, i}\right)$, which is a monotonically increasing function of the normalized Euclidean distance $D_{j, i}$ between sites $i$ and $j$. To normalize the distance between any two sites, one can divide this distance by the mean separation of the system sites. One can geometrically realize the mean site separation, if imagine a $d$-dimensional hypercube with length edges equal $L$ delimiting a system with $N$ sites. To each site one has to associate an also $d$-dimensional hypercube with length edges given by the mean site separation $\ell$. Now, if these hypercubes do not overlap, then we may write $N \ell^{d}=L^{d} \Rightarrow \ell=L N^{-1 / d}$. Thus, the normalized Euclidean distance is

$$
D_{j, i}=\frac{N^{1 / d}}{L}\left\{\sum_{k=1}^{d}\left[x_{i}^{(k)}-x_{j}^{(k)}\right]^{2}\right\}^{1 / 2} .
$$

In the thermodynamical limit, $N \rightarrow \infty$, this procedure preserves the constant site density as the system dimensionality varies, and makes it possible to compare systems with distinct dimensionalities.

To move on this landscape, the walker strategy based in the cost function $E\left(D_{j, i}\right)$ follows a probability density function (PDF) that depends on the inverse of the formal temperature, $T>0$, and the walker memory, $\mu \geq 0$. The inverse of a formal temperature $T$ is the stochastic parameter $\beta=1 / T$ and as $\beta$ decreases, long hops are favored. The memory generates a selfavoiding window with the last $\mu$ visited sites, which are forbidden to revisitation in the next $\mu$ steps. Thus, the PDF is given by:

$$
W_{j \leftarrow i}=\frac{e^{-\beta E\left(D_{j, i}\right)}}{\sum_{k=1}^{\prime N} e^{-\beta E\left(D_{k, i}\right)}},
$$

the summation $\sum^{\prime}$ excludes the forbidden sites by $\mu$. The denominator is known by normalization coefficient $Z_{i}^{(\beta, \mu)}$.

It is interesting to note that this probability privileges local exploration when $T \rightarrow 0(\beta \rightarrow \infty)$ and for $T=0$ the DTW is recovered. Now, when $T \rightarrow \infty(\beta \rightarrow 0)$ the walker can visit all permitted sites with the same probability $W_{j \leftarrow i}=1 /(N-\mu)$, for any allowed $j$. Thus, in which $T$ and $\mu$ one can expect the transition, between these localized and extended explorations, takes place?

In the following we show that a glass transition occurs at a sharp critical temperature for a specific cost function in a system 
with arbitrary dimensionality and $\mu=0$. Also, we stress the dificulties in the analytical calculations in one-dimensional systems but now with $\mu=1$. Finally we present our preliminary numerical results for one-dimensional systems when both temperature and memory vary.

\subsection{Lazy Tourist in Systems with Arbitrary Dimensionality: Glass Transition}

Martinez et al. [3] have analyzed stochastic walkers with memory $\mu=0$ moving on $d$-dimensional systems. This study focuses on a surprising connection between two apparently disparated fields: models of exploratory behavior $[5,6]$ and the statistical physics of glass transitions described by trap models [7, 8, 9, 10, $11,12]$. They show that a glass transition may appear when a walker explores a random landscape with localized resources. This exploration depends on a well-defined manner of the cost function $E\left(D_{j, i}\right)$ used to weigh the possible movements.

For each system dimension $d$, there is one specific cost function that enables a glass transition between the localized and extended exploratory behavior. The order parameter is the mean residence time $\left\langle t_{r}(\beta)\right\rangle$, i.e., the mean time that the walker spends on a site. Although any site has a finite trapping time, the average residence time $\left\langle t_{r}\right\rangle$ diverges below a finite stochasticity level $T_{d}$. In this case, the system falls into an out-of-equilibrium regime and aging phenomena appear.

To compute $\left\langle t_{r}(\beta)\right\rangle$, one may consider discrete time steps. The probability of a walker to remain in a given site $a$ is

$$
p_{a}(\beta) \equiv W_{a \leftarrow a}=\frac{1}{Z_{a}^{(\beta, 0)}}
$$

and the probability that the walker leaves site $a$ is $q_{a}(\beta)=$ $1-p_{a}(\beta)$. Calculations become easier if the distances are relabeled according to the ordering with respect site $a$, so that the nearest neighbor of site $a$ is at $D_{1}^{(a)}$, the second nearest neighbor is at $D_{2}^{(a)}$ and so forth. Then, the normalization coefficient may be written as

$$
Z_{a}^{(\beta, 0)}=1+\exp \left[-\beta E\left(D_{1}^{(a)}\right)\right] \sum_{j=1}^{N-1} \exp \left[-\beta \Delta_{j}^{(a)}\right],
$$

where $\Delta_{j}^{(a)}=E\left(D_{j}^{(a)}\right)-E\left(D_{1}^{(a)}\right)$.

Given that at $t=0$, the walker is at site $a$, the probability the walker remains there till time $t$ and leaves this site at $t+1$ is given by the geometric distribution (the first failure after $t$ successes): $P_{\beta}(t)=p_{a}^{t}(\beta) q_{a}(\beta)$. The residence (trapping) time associated to site $a$ is defined as the expected time:

$$
\begin{aligned}
t_{r}^{(a)}(\beta) & =\sum_{t=0}^{\infty} t p_{a}^{t}(\beta) q_{a}(\beta)=\frac{p_{a}(\beta)}{\left[1-p_{a}(\beta)\right]} \\
& =\exp \left[\beta E\left(D_{1}^{(a)}\right)\right]\left\{1+\sum_{j=2}^{N-1} \exp \left[-\beta \Delta_{j}^{(a)}\right]\right\} .
\end{aligned}
$$

The mean residence time for one realization of site distribution is $t_{r}(\beta)=\sum_{a=1}^{N} t_{r}^{(a)}(\beta) / N$ and the average over the disorder leads to

$$
\begin{aligned}
\left\langle t_{r}(\beta)\right\rangle= & \int d D_{1} \ldots d D_{N-1} P\left(D_{1}, \ldots, D_{N-1}\right) \\
& \times \frac{t_{1}(\beta)}{1+\sum_{j=2}^{N-1} e^{-\beta \Delta_{j}}},
\end{aligned}
$$

where $t_{1}(\beta) \equiv e^{\beta E\left(D_{1}\right)}$.

To calculate $\left\langle t_{r}(\beta)\right\rangle$, one needs to average over the full probability density distribution of neighbor distances $P\left(D_{1}, \ldots\right.$, $\left.D_{N-1}\right)$. This task is quite difficult since it takes the boundaries into account. Therefore, to remove the dependence on the boundaries one takes the thermodynamical limit $N \rightarrow \infty$. Although one cannot factorize the joint probability of the ordered distances $\left(D_{1}<D_{2}<\cdots<D_{N}\right)$, to continue the calculation one can make an approximation by considering only the effect on the trapping time of hops to the first neighbor. Here, we emphasize that this choice is due to two reasons:

1. the memory allows hops to the first neighbors, even the last visited one; and

2. in the limit of low temperature $(\beta \rightarrow \infty)$ the movements to nearest sites are greatly favored.

Thus, $\Delta_{N-1}>\cdots>\Delta_{2}>E\left(D_{1}\right)$ and the term $\sum_{j=2}^{N-1} e^{-\beta \Delta_{j}} \ll 1$ may be neglected in Eq. 4 and only the nearest neighbor distance PDF $P\left(D_{1}\right)$ is relevant, so that:

$$
\left\langle t_{r}(\beta)\right\rangle \cong\left\langle t_{1}(\beta)\right\rangle=\int_{0}^{\infty} d D_{1} P\left(D_{1}\right) e^{\beta E\left(D_{1}\right)} .
$$

It is possible to show numerically that $t_{1}(\beta)$ diverges with the same exponent and at the same $\beta$ as the exact $t_{r}(\beta)$ [3]. For a spatial Poisson process, the first neighbor probability density function is simply: $P\left(D_{1}\right)=S_{d}\left(D_{1}\right) e^{-V_{d}\left(D_{1}\right)}$, where we call the attention that $V_{d}(R)=A_{d} R^{d}$ is the volume of a hypersphere of radius $R$ and $S_{d}(R)=A_{d} d R^{d-1}$ is its surface. The geometrical factor $A_{d}=\pi^{d / 2} / \Gamma(d / 2+1)$ is the volume 
of an unitary radius hypersphere and $\Gamma(z)$ is the gamma function. Thus,

$$
\left\langle t_{1}(\beta)\right\rangle=A_{d} d \int_{0}^{\infty} d D_{1} D_{1}^{d-1} e^{\beta E\left(D_{1}\right)-A_{d} D_{1}^{d}} .
$$

Let us now focus our discussion on the cost function $E(D)$. If one considers $D$ being a distance, then one must expect that $E(D)$ is a monotonically increasing function of $D$, since the longer the distance, the more expensive to cover the trajectory. There are several ways that a function be a monotonically increasing function of its argument, for instance it can be a power law or exponential, or a generalized function that includes this situation [13]. In these cases the function increases indefinitively, nevertheless there are situations where the function increases up to a maximum value. Examples of this situation are the cumulative function (of probability density functions) in probability theory. Again, these functions can be subdivided in two categories, the ones which present an inflection point and others which do not present an inflection point. For instance, see the review of these functions presented in the context of population dynamics (or growth) models [14].

To continue, we choose the power-law form for the cost function: $E\left(D_{j, i}\right)=D_{j, i}^{\alpha}$, where $\alpha$ is an adjustable exponent. For differents value of $\alpha$ different behaviors can be examined:

- $\alpha<d$ : the $\left\langle t_{1}(\beta)\right\rangle$ is finite for any $\beta$ value, so that, for long times, the system is subdiffusive.

- $\alpha>d$ : the $\left\langle t_{1}(\beta)\right\rangle$ is always divergent and the system is superdiffusive.

- $\alpha=d$ : the more interesting situation appears, the residence time and the tail of the first neighbor distance distribution compete and a glass transition appears at finite value of $\beta$ :

$$
\left\langle t_{1}(\beta)\right\rangle= \begin{cases}1 /\left(1-\beta / A_{d}\right) & \left(\beta<A_{d}\right) \\ \infty & \left(\beta \geq A_{d}\right) .\end{cases}
$$

In this case, the mean residence time is finite only for $\beta<A_{d}$ and diverges at $\beta_{d}=A_{d}$, namely, until $\beta$ assumes the volume of a unitary hypersphere in $d$-dimensions the walker can explore the medium, after that the system entries in a non-equilibrium regime and aging phenomena emerge.

The residence time PDF as function of $\beta$ shows a powerlaw tail and a glass transition appears when the cost function
$E\left(D_{j, i}\right)=D_{j, i}^{d}$ is used. Notice however that these results arise from the particular distribution of sites used (Poisson process) and a power law cost function. We call the attention of our readers that other scenarios can be envisaged. For instance, when correlation is introduced among the sites. Further, future studies should consider other possibilities of cost functions, such as the generalized ones, which would bring more richness to the parameter space. For example, a distribution of sites where the distance PDF has a power-law tail.

These studies reveal us that a glass transition appears when the walker has no memory. Below, we see analytical results when the walker is prohibited to remain in the same site $\left(W_{a \leftarrow a}=0\right)$, that is, walker has memory $\mu=1$.

\subsection{Memoryless Tourist in One-dimensional Systems}

Let us now consider that the walker has memory $\mu=1$. Thus the walker cannot remain in the same site, and hops to one of its $n$ neighboring sites with a hop probability that depends on the distance $D_{j, i}$ between sites $i$ and $j$ according to a cost function $E\left(D_{j, i}\right)$. To examine this particular STW, Risau-Gusman et al. [4] have described the disordered landscape on a graph with ordered neighborhood. Then, all $N$ sites represent $N$ connected nodes and the probability of hops $W_{j \leftarrow i}$ provides a weight of the link between nodes $i$ and $j$. When hops occur in this graph, the network structure forms a directed graph. So, this graph presents interesting sets of nodes that we call sinks (i.e., sets with sites without outgoing links to sites outside the set), and sources (i.e., sets with sites without incoming links from sites outside the set).

The walk dynamics shows that for low temperature, the walker always goes to the nearest neighbor, eventually being trapped in a two-cycle when a pair of reciprocally nearest neighbors is found. We call this pair of mutually nearest neighbors of couples. The couples play the role of sites in the $\mu=0$ situation. However, for $T>0$, the two-cycles are no longer stable and can be characterized by a distribution of trapping times $P\left(t_{r}\right)$. From this distribution, one can compute the mean trapping time in twocycles using the joint distributions of distances for the spatial Poisson process.

Consider a cost function that permits a glass transition in this sites configuration. As discussed previously, an interesting cost function for one-dimensional systems is a linear function $E\left(D_{j, i}\right)=D_{j, i}$, since it may give rise to a crossover between different exploration regimes.

As in the preceding section, we order the distance according to couples. Now, let $a$ and $b$ be the sites of a couple, 
$D_{0}=D_{b, a}$ their pair distance, and $D_{j, a}\left(D_{j, b}\right)$ the distance from $a(b)$ to its $(j+1)$ th nearest neighbor. We can write the probability of hop from $a$ to $b$ and vice versa using (2):

$$
W_{b \leftarrow a}=\frac{e^{-\beta D_{0}}}{Z_{a}^{(\beta, 1)}}, \quad W_{a \leftarrow b}=\frac{e^{-\beta D_{0}}}{Z_{b}^{(\beta, 1)}} .
$$

Notice that these probabilities in general are different, since the passage seen from site $a$ is different from the one seen from site $b$.

Suppose that at $t=0$ the walker is at site $a$. The probability that the walker remains inside the couple for $t$ steps and then leaves it is $P_{e}(t)=\left(p_{a} p_{b}\right)^{t / 2} q_{a}$, for even values of $t$ and

$$
P_{o}(t)=\left(p_{a} p_{b}\right)^{(t-1) / 2} p_{a} q_{b}
$$

for odd values of $t$, with

$$
q_{a}=1-p_{a} \quad \text { and } \quad q_{b}=1-p_{b} .
$$

Since the walk may start either at site $a$ or site $b$, the probabilities that the walker remains in the couple up to time $t$ are $P_{e}(t)$ and $P_{o}(t)$. The expected residence time for this couple is:

$$
t_{r}=\frac{p_{a} p_{b}+\left(p_{a}+p_{b}\right) / 2}{1-p_{a} p_{b}} .
$$

Since one needs to include the bounds into account in order to obtain its characteristic diverging behavior, it is not possible to obtain $\left\langle t_{r}(\beta)\right\rangle$ through a straight forward analytical calculation. The strategy is to calculate in two extreme cases, which are physically intuitive and study their divergence. In the limit of low temperature, an approximation is the symmetrical approach where the distance from both members of the couple to their second neighbor is very larger and one can assume that this distance are equal for both sites $a$ and $b$; and the normalization coefficient are equal too $\left(Z_{a}^{(\beta, 1)}=Z_{b}^{(\beta, 1)}\right)$. Another approximation, in this limit, is the asymmetrical approach, where the distance $D_{1, a}$ is different from the distance $D_{1, b}$. Supposing that $D_{1, b} \gg D_{0}$, then the walker cannot leave the couple from site $b$.

From these approximations, the average residence time assume finite values when $\beta<\beta_{1}=2$, and diverges when $\beta=\beta_{1}=2$. Therefore, the system shows the behavior ergodic for high temperature $\left(\beta<\beta_{1}\right)$ and non-ergodic for low temperature, when $\beta \geq \beta_{1}$.

The critical temperature obtained here is the same as in the $\mu=0$ situation. One can understand this noticing that most of the 2/3 of the $N$ points form couple (mutually nearest neighbors) $[15,16]$ so that one can neglect the effect of $1 / 3$ of the
$N$ that form the transient in DTW. Considering one the couples ( $2 N / 3$ points) and now counting each time step as two moves (time renormalization) one obtain the same situation for $\mu=1$ as $\mu=0$, what explains the fact that both systems have the same critical temperature (the details are irrelevant).

What makes much more difficult to deal with $\mu \geq 2$ in STW is that in the determistic case, the system does not present a characteristic cycle period, but a whole spectrum [17], which inhibits time renormalization as done in the $\mu=1$ case.

\section{ONE DIMENSIONAL SYSTEMS AND ARBITRARY MEMORY VALUES}

The previous section describes the STW and reveals that one may find a glass transition at a critical temperature for $\mu=0$ in arbitrary system dimension or $\mu=1$ in one-dimensional systems. Now, we attempt to understand the effect of memory in the critical temperature. Before treating the problem analytically, we have implemented the STW algorithm in a computer and run it in a computer cluster. Our first numerical results for one-dimensional systems show the change in the system behavior when glass transition occurs.

\subsection{Algorithm}

On a unitary segment line, $N$ sites coordinates are independent and identically distributed (i.i.d.). The distance matrix $\left(D_{N \times N}\right)$ is then computed using the normalized Euclidean distance among all pairs of sites $i$ and $j$. In this case, the mean site separation is $\ell=1 / N$. To obtain the probability table, we have used the same cost function that enabled the glass transition in Section 2, $E\left(D_{j, i}\right)=D_{j, i}^{d}$, for one-dimensional system $d=1$. Several tables have been calculated for different temperature values ranging from 0.20 to 50.0 at different temperature steps.

With open boundary conditions $(\mathrm{OBC})$ several walks have been performed with memory varying from 0 to 25 . Since we hold that when the walker is able to visit all sites then his exploration is extended and the system display an ergodic behavior. For fixed number of walker steps, our goal was observe in which bandwidth of $T$ and $\mu$ the mean visitation rate of distinct sites changes radically. Thus, at each walker step, if a non-visited site were visited, then the mean visitation rate was incremented.

\subsection{Numerical Results}

In this way, our results show that for low temperatures, the mean visitation rate of distinct sites changes in a bandwidth of $\mu$ near the critical memory $\left(\mu_{1}\right)$, as in DTW. As one can behold in 


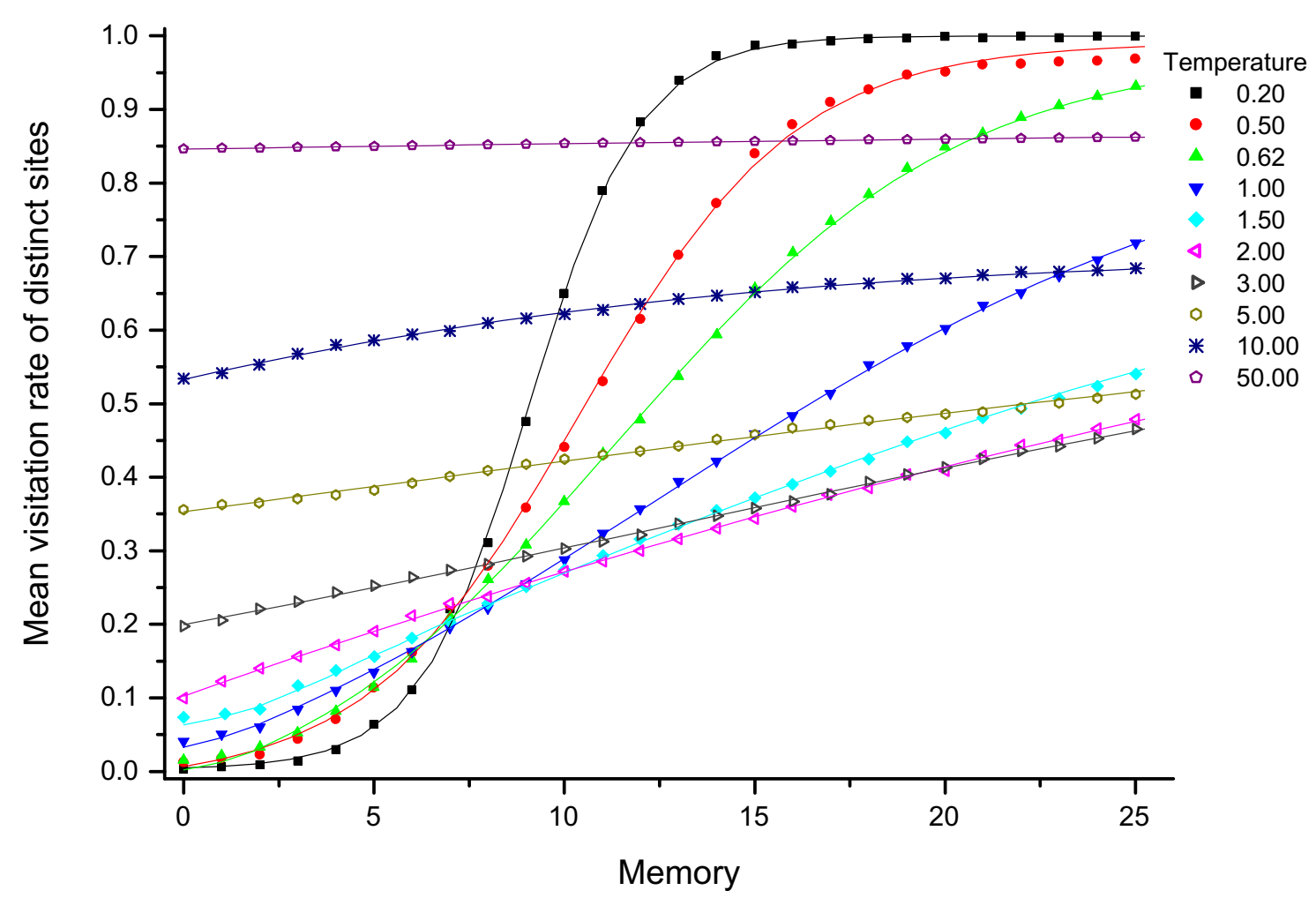

Figure 1 - Mean visitation rate of distinct sites versus memory, for ten temperatures. One can notice that the mean rate behavior drastically changes as the temperature increases.

the Figure 1, as the temperature increases, the dependence with memory decreases.

We can conclude that for one-dimensional systems, the STW exhibits dependence with memory untill the system reaches the critical temperature, after that the dependence with memory vanishes.

\section{CONCLUSIONS}

These results lead us to hold that this problem exhibits two distinct regimes: non-ergodic, where the walker can be trapped in localized exploration; and ergodic, where the walker can explore the whole medium. For instance, if a walker wants to find unvisited feeding sites, he needs to extend his exploration of the landscape. We conjecture that the efficiency of this kind of exploration is maximized when the cost function allows a glass transition. Then, the optimal exploratory process may occur at some temperature and walker memory above the glass line transition. Through simulations, we have already concluded that for one-dimensional systems, the STW exhibits dependence with memory untill the system reaches a critical temperature, after that the dependence with memory is vanished. Future works deal with analitycal studies about the system behavior as we take on different temperatures and memory for 1-dimensional systems and numerical studies for systems with high dimensionalities.

\section{ACKNOWLEDGEMENTS}

RSG(140420/2007-0), JMB(134461/2007-0) and ASM(303990/ 2007-4 and 476862/2007-8) acknowledges the Brazilian agency CNPq for support.

\section{REFERENCES}

[1] LIMA GF, MARTINEZ AS \& KINOUCHI 0. 2001. Deterministic Walks in Random Media. Phys. Rev. Lett., 87: 010603.

[2] STANLEY HE \& BULDYREV SV. 2001. Statistical physics - The salesman and the tourist. Nature (London), 413: 373-374.

[3] MARTINEZ AS, KINOUCHI 0 \& RISAU-GUSMAN S. 2004. Exploratory behavior, trap models, and glass transitions. Phys. Rev. E, 69: 017101.

[4] RISAU-GUSMAN S, MARTINEZ AS \& KINOUCHI 0. 2003. Escaping from cycles through a glass transition. Phys. Rev. E, 68: 016104. 
[5] VISWANATHAN GM, AFANASYEV V, BULDYREV S, MURPHY EJ, PRINCE PA \& STANLEY HE. 1996. Lévy flight search patterns of wandering albatrosses. Nature (London), 381: 413-415.

[6] VISWANATHAN GM, BULDYREV S, HAVLIN S, DA LUZ MGE, RAPOSOL EP \& STANLEY HE. 1999. Optimizing the success of random searches. Nature (London), 401: 911-914.

[7] BOUCHAUD J-P. 1992. Weak ergodicity breaking and aging in disordered-systems. J. Phys. I (France), 2: 1705-1713.

[8] DYRE JC. 1995. Energy master equation: a low-temperature approximation to Bassler's random-walk model. Phys. Rev. B, 51: 1227612294.

[9] MONTHUS C \& BOUCHAUD J-P. 1996. Models of traps and glass phenomenology. J. Phys. A, 29: 3847-3869.

[10] RINN B, MAASS P \& BOUCHAUD J-P. 2001. Hopping in the glass configuration space: subaging and generalized scaling laws. Phys. Rev. B, 64: 104417.

[11] BERTIN EM \& BOUCHAUD J-P. 2003. Subdiffusion and localization in the one-dimensional trap model. Phys. Rev. E, 67: 026128.
[12] DENNY RA, REICHMAN DR \& BOUCHAUD J-P. 2003. Trap models and slow dynamics in supercooled liquids. Phys. Rev. Lett., 90: 025503.

[13] ARRUDA TJ, GONZÁLEZ RS, TERÇARIOL CAS \& MARTINEZ AS. 2008. Arithmetical and geometrical means of generalized logarithmic and exponential functions: generalized sum and product operators. Phys. Lett. A, 372: 2578-2582.

[14] MARTINEZ AS, GONZÁLEZ RS \& TERÇARIOL CAS. 2008. Continuous growth models in terms of generalized logarithm and exponential functions. Physica A, 387: 5679-5687.

[15] KINOUCHI 0, MARTINEZ AS, LIMA GF, LOURENÇO GM \& RISAUGUSMAN S. 2002. Deterministic walks in random networks: an application to thesaurus graphs. Physica A, 315: 665-676.

[16] TERÇARIOL CAS \& MARTINEZ AS. 2005. Analytical results for the statistical distribution related to a memoryless deterministic walk: Dimensionality effect and mean-field models. Phys. Rev. E, 72: 021103.

[17] TERÇARIOL CAS \& MARTINEZ AS. 2008. The influence of memory in deterministic walks in random media: analytical calculation within a mean field approximation. Phys. Rev. E, 78: 031111. 